\title{
THE EFFECTS OF CHEWING GUM ON THE DRIVING PERFORMANCE OF EMERGENCY MEDICINE RESIDENTS AFTER OVERNIGHT SHIFT WORK
}

\author{
Maricel Dela Cruz, Muhammad Masood Khalid, Ahmed Mostafa, Jeffrey Foster, \\ Geoffrey Kaump, Rita G. McKeever, Michael I. Greenberg \\ Department of Emergency Medicine, Division of Medical Toxicology, \\ Drexel University College of Medicine \\ Philadelphia, PA, USA \\ Email: mdelacruz78@gmail.com
}

\begin{abstract}
Summary: This is a comparison study evaluating the influence of chewing gum on driving performance by computer simulation in emergency medicine residents doing overnight shift work. A total of eleven subjects were tested. Four simulations were randomized to each test subject at different points in the study. Data was analyzed comparing pre- and post-shift tests for each study group, as well as chewing gum versus non-chewing gum use during testing. Results showed no significant difference in lateral deviation, described as the root mean squared of lane departure measured in feet, or braking reaction time, defined as the time to break measured in seconds when triggered by a predetermined cue, in those using gum versus no gum. Between- and within-group differences were assessed by split-plot analysis measures analysis of variance (ANOVA). Our study showed statistical significance in that divided attention response time, designated as the time in seconds to perform a secondary task while driving, was longer in those driving with chewing gum versus without chewing gum $(\mathrm{p}<0.05)$. This pilot study serves as a potential foundation for further investigation into augmenting the driving performance of emergency medicine residents performing overnight shift work with chewing gum use.
\end{abstract}

\section{BACKGROUND}

Fatigued and drowsy driving are well-known human risk factors in motor vehicle collisions. The National Highway Traffic Safety Administration estimates that about 100,000 police-reported crashes result from driver fatigue annually, leading to roughly 1,550 deaths and 71,000 injuries per year (National Sleep Foundation, 2014). Circadian rhythm disruption and exhaustion are known occupational hazards for emergency medicine physicians as they have been associated with a decrement in shift performance as well as post-shift increases in motor vehicle collisions or near crashes (Kuhn et al., 2001; Lamberg et al., 2002; Steele et al., 1999; Machi et al., 2012).

\section{INTRODUCTION}

Survey studies analyzing the risk of motor vehicle collisions among emergency medicine residents have found an increase in reported motor vehicle collisions or near crashes after night shifts (Steele et al., 1999). Tornero et al., 2012, performed a battery of tests on twenty-five emergency medicine residents and found that in some cases, an emergency medicine night shift adversely affected certain skills needed for driving - specifically reaction time and visual-motor coordination. 
Chewing gum has been reported to increase alertness and to improve driving performance using the STISM3® Drive Simulator (Smith, 2009; Yoo et al., 2015). To our knowledge, no study has assessed the effect of chewing gum on driving performance in emergency medicine residents after an overnight shift in the emergency department. We evaluated the effects of chewing gum on driving performance after night shifts using the STISM3 ${ }^{\circledR}$ Driving Simulator. We divided the performance of the subjects into "chewing gum" and "non-chewing gum" arms and evaluated their driving performance under both conditions. Specifically, we analyzed driving performance using the following parameters: standard deviation of in-lane positioning (SDLP, i.e. "weaving"), described as the root mean squared of lane departure measured in feet, mean reaction time, defined as the time to break measured in seconds when triggered by a predetermined cue, and divided attention, designated as the time in seconds to perform a secondary task while driving. These parameters have been validated as comparable to on-road driving in studies evaluating driving ability.

\section{METHODS}

\section{Participants}

This was a comparison study evaluating the influence of chewing gum on driving performance by computer simulation in eleven emergency medicine residents (two female/nine male) doing overnight shift work. Subjects were recruited to complete one twenty-minute training session as well as two ten-minute pre-emergency department shift driving simulation sessions and two tenminute post-emergency medicine shift driving simulation sessions; subjects were compensated for their participation with a $\$ 60.00$ gift card.

Prior to starting the study, subjects were given a pre-training survey that gathered participant demographic information (i.e. age, sex, baseline driving ability, ethnicity, etc.) and self-reported level of fatigue. Subjects were provided written instructions prior to driving simulation training in order to avoid bias imparted by the trainer. Subjects underwent a validated twenty-minute driving simulation training and tutorial session on a computerized driving simulator. This session consisted of two different but similar driving simulations to accustom the subjects to the control of the device. This length of training has been validated in prior studies as the required time for most subjects to become comfortable with the simulator (Mayhew et al., 2011). The trainer was not allowed to further advise the subjects with the exceptions of clarifying the instructions. Once the training session was completed, the subjects were eligible to start the testing sessions.

Inclusion criteria consisted of the following: the current possession of a valid driver's license for a minimum of three years, a minimum of thirty minutes of driving per week, age eighteen years old or greater, and employment as a practicing third year emergency medicine resident working emergency department overnight shifts. Subjects were excluded from the study if they appeared visibly intoxicated, could not perform the driving simulator tasks, were fatigued at a level deemed inappropriate or unsafe for work or self-transportation home, or if they could not complete the entirety of the previously outlined requirements of the study. 


\section{Testing Device}

Driving simulators have become a recognized modality used to evaluate driving performance. Mayhew et al., 2011, compared the driving abilities of beginners, novices, and experienced drivers using both simulators and on-road testing. Their results showed that experienced drivers made the fewest mistakes, and errors made on the simulator were similar to mistakes made in onroad testing. In addition, the simulator we utilized in this study, the STISM3 ${ }^{\circledR}$ Drive Simulator, was employed in a study of 129 older adults comparing a driving simulator test with on-road testing using specific performance indices. A significant positive association between simulation driving and on-road testing was found which validated the use of this simulator (Lee, et al., 2003). These results support the use of the STISM3® Drive Simulator as a valid measure of driving performance. This system generates realistic roadway scenery, which is projected on a computer screen. Prefabricated simulations were created by Systems Technology Incorporated to test driving in various roadway settings. Simulations consisted of urban, suburban, rural, and highway driving, as well as specific driving situations such as passing slower vehicles. Auditory and tactile feedback is provided by speakers and steering wheel movement and includes the sound of the engine, braking, speeding, driving off the road, and passing traffic. Whenever a collision occurs, a broken windshield and the sound of braking glass are presented.

\section{Procedures}

This study was approved by the Drexel University institutional review board. Four of the premade simulations described above were randomized to test each subject at different points during the study. Computerized randomization was also used to determine when subjects were to be given chewing gum. Driving simulations used were pre-existing scenarios created by Systems Technology Incorporated on the STISM ${ }^{\circledR}$ Drive Simulator. In both pre- and post-shift driving simulation sessions, data collected included: standard deviation of lateral positioning ("weaving"), described as the root mean squared of lane departure measured in feet, divided attention, designated as the time in seconds to perform a secondary task while driving, and reaction time ("braking time"), defined as the time to break measured in seconds when triggered by a predetermined cue. Data was analyzed comparing pre- and post-shift tests for each study group, as well as chewing gum versus non-chewing gum tests. Between- and within-group differences were assessed using split-plot measures analysis of variance (ANOVA).

The emergency medicine residents at our facility have a progressive six-day shift work schedule. The third year resident schedule consists of eight-hour shifts; two early shifts (0700-1500), two middle shifts (1500-2300), followed by two overnight shifts (2300-0700). The subjects were then tested before and after each of two consecutive overnight shifts, with each simulation scenario lasting ten minutes. Prior to each simulation, subjects completed a pre- and post-shift questionnaire to assess their subjective level of fatigue.

\section{RESULTS}

A total of eleven subjects were tested (two female/nine male). After comparing pre-and post-shift tests for each study group, as well as chewing gum versus non-chewing gum tests, between- and within-group differences were assessed using split-plot measures analysis of variance (ANOVA). 
Mean divided attention response time was 1.95 seconds ( $\mathrm{SD}+/-0.27$ seconds) in the chewing gum group and 1.68 seconds ( $\mathrm{SD}+/-0.13$ seconds) in the non-chewing gum group. The average braking reaction time was 1.86 seconds ( $\mathrm{SD}+/-0.06$ seconds) in the chewing gum group and 1.81 seconds ( $\mathrm{SD}+/-0.06$ seconds) in non-chewing gum group. Lateral deviation, measured as the root mean squared of lane deviance was 0.90 (SD +/- 0.70) in gum users and $0.71(\mathrm{SD}+/-$ $0.05)$ in non-gum users.

Results showed no significant difference in lateral deviation, described as the root mean squared of lane departure measured in feet, or braking reaction time, defined as the time to break measured in seconds when triggered by a predetermined cue, in those using gum versus no gum or in pre- versus post-shift driving tests. Our study did show statistical significance in that divided attention response time was longer in those driving with chewing gum versus without chewing gum $(\mathrm{p}<0.05)$. See Figure 1.

\begin{tabular}{|l|l|c|c|}
\hline Response & Independent Variable & Test Statistic & PValue \\
\hline $\begin{array}{l}\text { Divided Attention } \\
\text { Response Accuracy }\end{array}$ & Gum vs. No Gum & $\chi^{2}(2)=3.26$ & 0.20 \\
\hline & Pre vs. Post & $\chi^{2}(2)=3.55$ & 0.17 \\
\hline Interaction & $\chi^{2}(6)=9.97$ & 0.13 \\
\hline $\begin{array}{l}\text { Divided Attention } \\
\text { Response Time }\end{array}$ & Gum vs. No Gum & $F(I, 3)=9.47$ & $<0.05$ \\
\hline & Interaction & $F(I, 3)=2.53$ & 0.21 \\
\hline
\end{tabular}

Figure 1. Divided Attention Response Accuracy and Divided Attention Response Time.

\section{DISCUSSION}

Chewing gum has been reported to increase alertness and to improve driving performance using the STISIM3 ${ }^{\circledR}$ Drive Simulator. We divided subjects into "chewing gum" and "non-chewing gum" arms and evaluated their driving performance under both conditions. We analyzed driving performance using the parameters: standard deviation of in-lane positioning (SDLP), described as the root mean squared of lane departure measured in feet, mean reaction time, defined as the time to break measured in seconds when triggered by a predetermined cue, and divided attention, designated as the time in seconds to perform a secondary task while driving. As mentioned earlier, these parameters have been validated as comparable to on-road driving in studies evaluating driving ability. Our study shows that among two parameters, lateral deviation and braking reaction time, chewing gum and pre- or post-shift times did not make a difference. However, divided attention response time was prolonged in those chewing gum versus those not chewing gum while driving with the simulator.

In comparison to other studies which show the possible improvement of cognitive function, enhancement in performance and increase in alertness with the use of chewing gum (Smith, 2009; Smith, 2010), our study showed no significant difference between the gum and non-gum 
users concerning shift work and driving performance in relation to standard deviation of in-lane positioning or mean reaction time. Furthermore, unlike this prior research (Smith, 2009; Smith, 2010), our study showed a decline in driving performance in regards to divided attention response time; the time in seconds to perform a secondary task while driving was prolonged before and after emergency department shift work with use of chewing gum versus no use of chewing gum. Task switching and divided attention may differ from the other recorded driving performance study items of standard deviation of in-lane positioning and braking reaction time as it introduces a third task, duty or undertaking, while lateral deviation and braking are directly related to the primary driving task at hand. The provided divided attention undertaking was entirely unrelated to the chief driving task, and may have introduced a newly learned, unfamiliar behavior that was more disruptive than natural for the common driver who may now be inherently accustomed to compensating for lane deviation or braking when required.

\section{CONCLUSION}

Antecedent research has shown that chewing gum can enhance performance of other tasks such as memory, recall, cognitive function, learning, mood, and physiology in stressed and nonstressed individuals (Smith, 2009; Smith, 2010; Rickman et al., 2013). Similar investigations have also shown increased alertness while driving with the use of chewing gum (Smith, 2009; Yoo et al., 2015), but limited information is found regarding driving performance and chewing gum use. In terms of subjective level of fatigue, all tested subjects did not feel more or less fatigued with or without chewing gum use, even though their driving performance measured with divided attention declined with chewing gum. This pilot study serves as a foundation for further investigation into improving the driving performance of emergency medicine resident physicians performing overnight shift work with chewing gum use. Further studies may be implicated to explore the implications of chewing gum use and driving performance safety.

\section{REFERENCES}

The National Sleep Foundation. (2014, November 2). Facts and Stats, Drowsy Driving Prevention Week. [Web post]. Retrieved from January 5, 2015 http://drowsydriving.org/about/facts-and-stats/.

Kuhn, G. (2001). Circadian rhythm, shift work, and emergency medicine. Annals of Emergency Medicine, 37, 88-98.

Lamberg L. (2002). Long hours, little sleep: bad medicine for physicians-in-training? The Journal of the American Medical Association, 287, 303-306.

LaSala G.S., McKeever R.G., Patel U., Okaneku J, Vearrier D.J., \& Greenberg M.I. (2015). Effect of single-dose Ginkgo biloba and Panax ginseng on driving performance. Clinical Toxicology, 53(2), 108-112.

Lee H.C., Cameron D., \& Lee A.H. (2003). Assessing the driving performance of older adult drivers: on-road versus simulated driving. Accident Analysis and Prevention, 35(5), 797803. 
Machi M.S., Staum M., Callaway C.W., Moore C., Jeong K., Suyama J., Patterson P.D., \& Hostler D. (2012). The relationship between shift work, sleep, and cognition in career emergency physicians. Academic Emergency Medicine: Official Journal of the Society for Academic Emergency Medicine, 19(1), 85-91.

Mayhew D.R., Simpson H.M., Wood K.M., Lonero L., Clinton K.M., \& Johnson A.G. (2011). On-road and simulated driving: concurrent and discriminant validation. Journal of Safety Research, 42(4), 267-275.

Smith A. (2009). Effects of chewing gum on mood, learning, memory, and performance of an intelligence test. Nutritional Neuroscience, 12(2), 81-88.

Steele M.T., Ma O.J., Watson W.A., Thomas H.A., \& Muelleman R.L. (1999). The occupational risk of motor vehicle collisions for emergency medicine residents. Academic Emergency Medicine: Official Journal of the Society for Academic Emergency Medicine, 6(10), 1050-1053.

Tornero C., Ventura A., Bourguet M., \& Poquet I. (2012). Evaluation of driving ability among residents after the duty shift. Accident Analysis and Prevention, 47(1), 182-183.

Yoo I., Kim E.J., \& Lee J.H. (2015). Effects of chewing gum on driving performance as evaluated by the STISM ${ }^{\circledR}$ Drive Simulator. Journal of Physical Therapy Science, 27(6), 1823-1825.

Smith A. (2010). Effects of chewing gum on cognitive function, mood and physiology in stressed and non-stressed volunteers. Nutritional Neuroscience, 13(1), 7-16.

Rickman S., Johnson A., \& Miles C. (2013). The impact of chewing gum resistance on immediate free recall. British Journal of Psychology. 104(3), 339-346. 